\title{
Distinctiveness of Disability Due to Psychiatric Conditions
}

\author{
Jagadisha Thirthalli $\cdot$ Chethan Basavarajappa
}

Received: 26 September 2014/ Accepted: 29 September 2014/Published online: 7 November 2014

(C) Springer India Pvt. Ltd. 2014

\section{Introduction}

The World Health Organization (WHO)'s International Classification of Functioning, Disability and Health (ICF) [1] has a number of fundamental principles, which reflect the contemporary 'bio-psycho-social' model of disability. Universality, parity and neutrality are examples of such principles. These principles are basically meant to dissociate the concept of disability from the health condition that could have caused the disability. This important paradigm shift would prevent labeling of persons with disabilities and any differential treatment of persons with disabilities due to different health conditions that they experience. Specifically, the ICF discourages the distinction based on 'physical' and 'mental' health conditions. This modern outlook towards disability has several advantages. Particularly in the case of persons experiencing psychiatric conditions, this approach goes a long way towards reduction of stigma and discrimination specifically attached with such conditions.

However, from the perspective of intervention and rehabilitation, a few distinctive features of disability due to psychiatric conditions need further deliberation. Here we highlight four issues, which are of relevance while planning and providing interventions for persons who suffer disabilities due to psychiatric conditions like schizophrenia, bipolar disorders, obsessive compulsive disorder, depression, etc.

J. Thirthalli $(\bowtie) \cdot$ C. Basavarajappa

Psychiatric Rehabilitation Services, Department of Psychiatry,

National Institute of Mental Health \& Neurosciences

(NIMHANS), Bangalore, Karnataka, India

e-mail: jagatth@yahoo.com
1. Need for continued clinical care In the ICF, the terms "medical treatment/care" and "medication" are suggested as interventions at the level of "impairment" and not under "activity limitation" or "participation restriction". "Rehabilitative therapy" is suggested as an intervention for the latter. This is typically applicable for physical health conditions, where the intensive medical/surgical treatments would be useful at the initial stages of the illness in order to minimize the disability; little medical intervention is warranted once disability per se has set in. However, in the case of disability due to psychiatric illnesses, continued care by clinicians including psychiatrists, psychologists, psychiatric social workers and nurses is almost indispensable. It is in this context that the boundary between "treatment" and "rehabilitation" becomes blurred. For example in persons with spinal injury, the neurosurgeon's intervention may limit disability, but after the procedure is over, the surgeon has minimal role in rehabilitation. In contrast, for most psychiatric disorders, continued care by professionals who provide "treatment" in the acute phase would be required for nearly indefinite period because of the following reasons:

(a) Discontinuation of psychotropic medications in most cases would result in repeated relapses, which contribute to disability. At the same time, at different stages through the course of the illness, modification in the type and dosage of medications would be necessary lest the medications per se add to the disability (like sedation, extra-pyramidal symptoms, obesity etc.).

(b) A number of symptoms, management of which is typically associated with "treatment", may 
add to disability; e.g., post psychotic depression, co-morbid substance use/anxiety disorder etc., in the case of schizophrenia. Hence "rehabilitation therapy" in the case of psychiatric disability would involve elements which are typically associated with "treatment" of the psychiatric disorders.

2. 'Rehabilitation' itself as 'treatment' The WHO has provided an umbrella term, disability, to represent impairment, activity limitation and participation restriction. Often, in the case of physical disabilities, "rehabilitation" does not address or affect impairment per se. Rather, rehabilitation is mostly geared towards alleviating activity limitation, and, to some extent, participation restriction. For instance, in the case of the visually challenged individuals, provision of a suitable gainful employment (e.g., as a music teacher) is not expected to improve his/her vision. Rather, it alleviates his/her participation restriction.

An interesting aspect of psychiatric disability is the fact that rehabilitation process has a potential to improve impairment per se. For instance, an individual afflicted with schizophrenia may be disabled due to certain symptoms (i.e., impairment) of the illness, like depression, demoralization, low self-esteem and poor social skills. If he/she is assisted to get a gainful employment (which is an intervention towards participation restriction), this 'rehabilitative' input itself could improve his/her self-esteem, reduce depression and also result in better social skills. Thus, rehabilitative efforts in those with disability due to psychiatric disorders can actually be therapeutic in that they tend to act at all levels of disability: impairment, activity limitation and participation restriction.

3. Role of insight In the contemporary field of rehabilitation, utmost importance is given to the personal autonomy of the individuals with disability. In the case of persons with physical disabilities, there is little debate about this issue. In contrast, it becomes a contentious issue in the case of psychiatric disabilities. At least in a significant proportion of persons with psychiatric disabilities, the very ability of thinking about one's autonomy and taking reasonable steps to exercise it would be compromised. For instance, a visually challenged person could be considered to be using his/her autonomy in an undisputable manner if he/she refuses any rehabilitative efforts that he/she prefers not to make use of. However, in contrast, a person with catatonic schizophrenia may resist attempts to treat him/her and continue to experience substantial disability because of the very fact that he/ she has the illness.

4. Dynamic nature of psychiatric disability In case of most physical disabilities the disability per se remains static. In contrast, in the case of psychiatric disabilities an individual goes through phases when he/she is profoundly disabled and when he/she is nearly free of any disabilities. There is lack of clarity regarding the status of rehabilitation and welfare benefits for those with psychiatric disability when they are in a "nondisabled" phase. Withdrawal of support in the form of welfare benefits and rehabilitation efforts at this stage would be inappropriate and counterproductive. For instance, at least in a proportion of persons with mental illness in some countries, the person might use the disability pension to procure psychotropic medications, which helps him/her to keep disability to the minimum. Withdrawal of welfare benefits might result in them not being able to procure the medications resulting in relapse, thus exacerbating their disability. Such variations in the level of disability are not common in those with disability due to physical illnesses.

Evidently, disability secondary to psychiatric conditions has features that are distinctive in nature. While the issues regarding parity are undoubtedly relevant and progressive, one cannot be oblivious to these distinctive features of disability related to psychiatric conditions. We emphasize the need for deliberations at international level with regard to these issues. Further, given the influence that culture has on disability and its remediation, such deliberations should specifically involve stakeholders from diverse regional and cultural settings.

\section{Reference}

1. World Health Organization. Towards a common language for functioning, disability and health, ICF. Geneva: World Health Organization; 2002. 